\title{
PAIR-COUNTABLE, CLOSURE-PRESERVING COVERS OF COMPACT SETS
}

\author{
HENRY POTOCZNY \\ (Received 29 November 1983; revised 26 February 1986) \\ Communicated by J. H. Rubinstein
}

\begin{abstract}
In this paper, we prove the following results: (1) if a topological space $X$ has a pair-countable, closure-preserving cover of compact sets, then $X$ is locally paracompact at each point of $X$ and $X$ has a dense open subspace which is locally $\sigma$-compact. In addition, if $X$ is also collectionwise- $T_{2}$, then $X$ is paracompact. Locally paracompact is taken to mean that each point $X$ has an open set with paracompact closure.
\end{abstract}

1980 Mathematics subject classification (Amer. Math. Soc.): primary 54 D 18; secondary 54 D 45.

\section{Introduction}

In [8], Tamano asked whether or not a space which is the closure-preserving union of compact sets must be paracompact. It was shown in [4] that the answer is negative, an example being given there of a topological space which is the union of a closure-preserving family of finite sets, is completely regular and $T_{2}$, even has a basis consisting of open and closed sets, yet fails to be even normal, hence certainly not paracompact.

In a positive direction, it was shown in [5] that if a topological space $X$ has a point-countable closure-preserving cover of compact sets, then $X$ is paracompact, in fact, $X$ is the pairwise disjoing union of a family of open and closed $\sigma$-compact subsets.

(C) 1988 Australian Mathematical Society $0263-6115 / 88 \$ A 2.00+0.00$ 
As regards historical perspective, the following results are of interest.

(1) If a regular space $X$ is strongly collectionwise-normal with respect to compact sets, and has a closure-preserving cover by compact sets, then $X$ is paracompact.

(2) If a $T_{1}$ space has a closure-preserving cover by closed compact sets, it is metacompact.

The first is essentially shown in [6]: The phrase, "with respect to compact sets", is omitted, but that is all that is used in the proof. The second result was shown in [7], and independently, in [2].

The first result gives a much-needed perspective on Theorem 3 , since it makes the following question natural: If a regular space $X$ is strongly collectionwise- $T_{2}$, and has a closure-preserving cover by compact sets, is $X$ paracompact?

In this paper, we prove the following related results: (1) If a topological space $X$ has a pair-countable, closure-preserving cover of compact sets, then $X$ is locally paracompact at each point of $X$ and $X$ has a dense open subspace which is locally $\sigma$-compact. In addition, if $X$ is also collectionwise- $T_{2}$, then $X$ is paracompact. Locally paracompact is taken to mean that each point $X$ has an open neighborhood with paracompact closure.

Definitions. A family $\mathscr{F}$, of sets, is said to be pair-countable provided that for each two distinct points $x$ and $y$, at most countably many members of $\mathscr{F}$ contain both $x$ and $y$. A family $\mathscr{F}$ of sets is of countable order at $x$ if $x$ is an element of at most countably many members of $\mathscr{F}$. Otherwise, $\mathscr{F}$ is said to be of uncountable order at $x$.

In what follows, regular is understood to include $T_{2}$.

Lemma 1. Let $x$ be a topological space and let $\mathscr{F}=\{F(\alpha) \mid \alpha \in A\}$ be a closure-preserving closed cover of $A$ by compact sets. For each $x \in X$, let $K(x)=$ $\{y \in X \mid$ if $y \in F(\alpha)$, then $x \in F(\alpha)\}$. Then

(i) $K(x)=X-\bigcup\{F(\alpha) \mid x \notin F(\alpha)\}$,

(ii) $K(x)$ is open, and if $y \in K(x)$, then $K(y) \subset K(x)$,

(iii) if $\mathscr{F}$ is of countable order at $x \in X$, then $K(x)$ has $\sigma$-compact closure.

LemMA 2. Let $X$ be a regular space with a point $x$ such that the complement of every open neighborhood of $x$ is paracompact. Then $x$ is paracompact.

Proof. The proof is straightforward and is left as an exercise.

LEMMA 3. Let $X$ be a regular space with a closure-preserving cover $\mathscr{F}=\{F(\alpha) \mid \alpha$ $\in A\}$, of compact sets. If $\mathscr{F}$ is of countable order at all but at most one point, then $X$ is paracompact. 
Proof. Let $W$ be an open set about $X$. The family $\{F(\alpha) \cap(X-W) \mid \alpha \in A\}$ is a closure-preserving, point-countable cover of $X-W$ by compact sets. By Corollary 2 of [2], $X-W$ is the disjoint union of open and closed $\sigma$-compact subspaces, hence is certainly paracompact. Paracompactness of $X$ follows from Lemma 2.

LEMMA 4. Let $X$ be a regular topological space and let $\mathscr{F}=\{F(\alpha) \mid \alpha \in A\}$ be a pair-countable, closure-preserving cover of $X$ by compact sets. Let $M$ be the set of all points of uncountable order. Then $M$ is closed and every subset of $M$ is closed.

Proof. Let $N$ be a subset of $M$, and let $x$ be a point of $X-N$. Consider the open set $K(x)$. If $K(x) \cap N$ is not empty, let $p \in K(x) \cap N$. Then $p$ is certainly of uncountable order. Moreover, by Lemma 1, if $p$ appears in a member of $\mathscr{F}$, so does $x$. But then $p$ and $x$ appear together in an uncountable number of members of $\mathscr{F}$, and this violates the pair-countability of $\mathscr{F}$. Hence $K(x) \cap N=\varnothing$, and $N$ is seen to be closed.

THEOREM 1. Let $X$ be a regular topological space and let $\mathscr{F}=\{F(\alpha) \mid \alpha \in A\}$ be a pair-countable, closure-preserving cover of $X$ by compact sets. Then $X$ is locally paracompact, that is, each point $X$, of $X$, has an open set with paracompact closure.

Proof. If $x$ is a point of countable order, then by Lemma $1, x$ has an open neighborhood with $\sigma$-compact closure which, in the presence of regularity, implies the existence of the Lindelö property, hence implies paracompactness. If $x$ is a point of uncountable order, then consider the open set $K(x)$. This is an open set whose closure may contain some points of $M$, the set of points of uncountable character. By the previous lemma, however, $M-\{x\}$ is closed, and since $X$ is regular, there is an open set $V$ such that $x \in V \cap \bar{V}$ and $\bar{V} \cap(M-\{x\})=\varnothing$. Now the family $\{\bar{V} \cap F(\alpha) \mid \alpha \in A\}$ is easily seen to be a closure-preserving pair-countable cover of $\bar{V}$ by compact sets. Moreover, $\bar{V}$ clearly contains only one point of uncountable order, $x$ itself, so that Lemma 3 applies, and $\bar{V}$ is seen to be paracompact.

THEOREM 2. Let $X$ be a regular topological space. Let $\mathscr{F}=\{F(\alpha) \mid \alpha \in A\}$ be a closure-preserving pair-countable cover of $X$ by compact sets. Then $X$ has a dense open subspace which is locally $\sigma$-compact.

Proof. Let $M$ be the set of points of uncountable order. Let $Y=(X-M) \cup$ $\{x \mid x \in M,\{x\}$ is open $\}$. Then $Y$ is a dense open subspace of $X$, and is locally $\sigma$-compact. To see that $Y$ is open, note that if $p$ is of countable order so is every 
point of $K(p)$, whence $p \in K(p) \subset Y$; and certainly, $\{x \mid x \in M,\{x\}$ is open $\}$, is an open set.

To see that $Y$ is locally $\sigma$-compact, it clearly suffices to show that for each $p \in X-M, p$ has an open set with $\sigma$-compact closure in $Y$. By Lemma 1 , each point $p$ has the open neighborhood $K(p)$ whose closure in the entire space $X$, is $\sigma$-compact. But since $X$ is regular, there is an open set $V$ such that $p \in V \subset \bar{V} \subset$ $Y$. Then $K(p) \cap V$ contains the point $p$ and has $\sigma$-compact closure in $Y$.

To see that $Y$ is a dense subspace of $X$, notice that only points of $M$ are candidates to be limit points of $Y$. Let $x \in M$, and suppose $X$ has an open set $V$ which does not meet $Y$. By Lemma 4, M-\{x\} is closed so that there is an open set $W$ such that $x \in W$, and $W \cap(M-\{x\})=\varnothing$. But then $v \cap W$ is evidently $\{x\}$, so that $x \in Y$ after all.

Definition. A topological space $X$ is said to be collectionwise- $T_{2}$ provided that for each discrete family of singleton sets, there is a pairwise disjoint family of open sets, each containing one of the singletons.

Definition. A topological space $X$ is said to be strongly collectionwise- $T_{2}$ provided that if $\{\{x(\alpha)\} \mid \alpha \in A\}$ is a discrete family of singletons, then there is a discrete family $\{V(\alpha) \mid \alpha \in A\}$, of open subsets of $X$ such that for each $\alpha \in A$, $x(\alpha) \in V(\alpha)$.

THEOREM 3. Let $X$ be a regular topological space. Let $\mathscr{F}=\{F(\alpha) \mid \alpha \in A\}$ be a closure-preserving pair-countable cover of $X$ by compact sets. If $X$ is also strongly collectionwise- $T_{2}$, then $X$ is paracompact.

ProOf. Let $M$ be the set of points of uncountable order. Then, by Lemma 4, the set of singletons $\{\{x\} \mid x \in M\}$ is a discrete family.

Now let $\mathscr{B}=\{B(x) \mid x \in M\}$ be a discrete family of open subsets of $X$ such that for each $x \in M, x \in B(x)$. To see that $X$ is paracompact, let $\mathscr{V}$ be an open cover of $x$. For each $x \in M$, let $V(x)$ be a member of such that $x \in V(x)$. For each $x \in M$, let $W(x)=B(x) \cap V(x)$. Then the family $\{W(x) \mid x \in M\}$ is a discrete family of open subsets of $X$, each set containing the point on which it is indexed. Since it is a discrete family, it is certainly closure-preserving so that if we set $W=\bigcup\{W(x) \mid x \in M\}$, then $\bar{W}=\overline{U\{W(x) \mid x \in M}\}=\bigcup\{\overline{W(x)} \mid x \in M\}$ which is a subset of $V=\bigcup\{V(x) \mid x \in M\}$. We now have $M$, the set of points of uncountable order inside the open set $W$, and $\bar{W} \subset V$. We may now observe that $X-W$ is a closed subset of $X$, so that $\{F(\alpha) \cap(X-W) \mid \alpha \in A\}$ is pair-countable, closure-preserving cover of $X-W$ by compact sets. Moreover, each point is of countable order, so that $X-W$ is the disjoint union of open and closed $\sigma$-compact subspaces, hence is paracompact. The proof may now proceed along lines similar to those of Lemma 2. 
Corollary. Let $X$ be a topological space and let $\mathscr{F}=\{F(\alpha) \mid \alpha A\}$ be $a$ closure-preserving, pair-countable family of compact sets which covers $X$. The following are equivalent

(i) $X$ is paracompact,

(ii) $X$ is normal and collectionwise- $T_{2}$.

(iii) $X$ is regular and strongly collectionwise- $T_{2}$.

The implications (i) $\rightarrow$ (ii) $\rightarrow$ (iii) are well known for topological spaces in general, while (iii) $\rightarrow$ (i) is the content of Theorem 3 .

Examples. An example to illustrate the main results is the Pixley-Roy space $\mathscr{F}(R)$, on $R$, and its subspaces, (see [3]). Elements are finite non-empty subsets of $R$. Basic open sets are of the form $[B, U]=\{C \in \mathscr{F}(R) \mid B \subset C \subset U\}$, where $U$ is an open subset of $R$ such that $B \subset U$. The space has a closure-preserving cover by the finite sets $\{\mathscr{P}(B) \mid B \in \mathscr{F}(R)\}-\{\varnothing\}$, but does not, however, have a dense paracompact subspace, hence "pair-countable" cannot be omitted from Theorem 3. On the other hand, it has a subspace $\{B|B \subset R, 0 \leqslant| B \mid<2\}$, which does have a pair-countable closure-preserving cover by finite sets. The subspace, however, has no point-countable closure-preserving cover by compact sets. In fact, this subspace is simply "Heath's Tangent $V$ Space" (see [1]).

This latter space also serves as an example to show that "strongly collectionwise- $T_{2}$ " cannot be omitted from Theorem 3 .

\section{References}

[1] R. Heath, 'Screenability, pointwise paracompactness, and metrization of Moore spaces', Canad. J. Math. 16 (1964), 763-770.

[2] Y. Katuta, 'On spaces which admit closure-preserving covers by compact sets', Proc. Japan Acad. 50, No. 10 (1974).

[3] D. Lutzer, 'Pixley-Roy topology', Topology Proc. 3 (1978), 139-158.

[4] H. Potoczny, 'A nonparacompact space which admits a closure-preserving cover of compact sets', Proc. Amer. Math. Soc. 32 (1972), 309-311.

[5] H. Potoczny, 'On a problem of Tamano', Fund. Math. 75 (1972), 29-31.

[6] H. Potoczny, Closure-preserving families of compact sets, General Topology Appl. 3 (1973), 243-248.

[7] H. Potoczny and H. Junnila, 'Closure-preserving families and metacompactness', Proc. Amer. Math. Soc. 53 (1975), 523-529.

[8] H. Tamano, 'A characterization of paracompactness', Fund. Math. 72 (1971), 189-201.

Department of Mathematics and Computer Science

U.S. Air Force Institute of Technology

Wright-Patterson, Ohio 45433 